\title{
AFTERWORD: A CALL TO ACTION ON COVID-19 RESPONSE IN ASSISTED LIVING/RESIDENTIAL CARE COMMUNITIES
}

The year of publication, 2020, for this second edition of Assisted Living Administration and Management: Effective Practices and Model Programs in Elder Care will be remembered as one influenced by an unrelenting coronavirus and social unrest. Despite statewide shelter-in-place, social distancing, face mask and handwashing guidelines, the unprecedented COVID-19 global pandemic has disproportionately affected older Americans, who have higher rates of chronic health conditions, which has led to severe illness and death. During the COVID-19 public health crisis, the social unrest did not focus on the plight of the elderly, who suffered from not-so-subtle discrimination stemming from ageism (bias based solely on advanced age), double jeopardy (ageism and racial bias) or multiple jeopardy (double jeopardy and additional bias). Instead, the social unrest has focused mainly on multiple reluctant parties who have failed to social distance properly; the Black Lives Matter and "my body, my choice" demonstrations among other community protests and social events potentially have added to COVID-19 cases and deaths.

In May 2020, the Associated Press reported that a Northern California city official, Ken Turnage, was ousted after he posted on social media that sick, old, and injured people should be left to meet their "natural course in nature" during the coronavirus pandemic (https:/www .nbcnews.com/politics/politics-news/california-city-official-ousted-over-his-pandemicremarks-n1198716). Although Turnage is entitled to his freedom of speech, his comments proposed that the elderly are expendable for the good of younger people. This misguided thinking was alluded to in a conversation about "Out With the Old: Coronavirus Highlights Why We Need New Names for Aging” with Caroline Cicero and Paul Nash, instructional associate professors of gerontology at the University of Southern California (https://theconversation.com/ out-with-the-old-coronavirus-highlights-why-we-need-new-names-for-aging-131380).

Cicero and Nash talked about the marginalization of older Americans with the rise of COVID19. Some blame the elderly for the shelter-in-place guidelines, and some even say the elderly should be sacrificed for the good of the country. Nevertheless, the coronavirus affects young and old alike. Although hospitalization and mortality rates increase with age, the Centers for Disease Control and Prevention report that young adults take up more ICU beds than the very old. Optimistically, aging is something we will all experience. Yet ageism is possibly the last widely accepted form of social prejudice. Certainly, the World Health Organization (WHO) believes ageism may be more pervasive than sexism or racism. Research clearly shows negative attitudes about aging when being older can harm your health and well-being, even impact your mortality. 
It is very disconcerting that the elderly experienced such prejudicial treatment during the COVID-19 pandemic without redress. Both the lack of compassion for, and ignorance about, the elderly population expressed by the actions of many (but not all) younger adults, who deliberately chose not to shelter-in-place, speak volumes. Making it worse, given the enhanced longevity of our aging society, seniors living in assisted living/residential care communities (skilled nursing facilities) feared that their lives were seen as expendable in the rush to reopen the country. Clearly, as a nation, we can do better to help educate and train younger Americans to better appreciate and care for our aging population. Studies have found that students benefit academically as well as socially from taking gerontology courses. Gerontology courses play an important role in building an inclusive intergenerational and multicultural society.

Yet, in order to educate and train people for meaningful advocacy and careers in aging, more colleges and universities must offer and require courses and programs in gerontology so that students may have opportunities to learn about the aged, aging processes, and elder care workforce needs for an aging world. These innovative gerontology courses and programs could help to: (a) emphasize the broad, interdisciplinary nature of issues that relate to and influence older adults; (b) provide students with the academic background, professional experience, and research capabilities necessary to pursue advanced and professional study pertaining to an aging population; and (c) prepare students for professional practice and leadership positions in the public and private sectors where gerontological knowledge is required. We urgently need to educate younger adults about aging and long-term care so that they may contribute to "age-friendly communities" that will better address health and social needs as well as individualized and coordinated services that promote independence and maximize "quality of life" for young and old alike.

In some states, such as California, there have been early legislative attempts to include gerontology education and geriatric training in the college curriculum. One such legislative bill was the California Integrated Elder Care and Involvement Act of 2002 (Senate Bill [SB] 953; Vasconcellos, Chapter 541, Statutes of 2002), which called for: (a) gerontology and geriatric training; (b) academic standards (Requested the California State University [CSU], University of California [UC] and California Community College [CC] systems to develop standards and guidelines for the biological, social, and psychological aspects of aging for professional degree programs at both the bachelor and graduate level, in which the health and welfare of older adults is paramount); and (c) professional licensure requirements. Another legislative bill was Gerontology Service Delivery Personnel: Training (Assembly Bill [AB] 2202; Alquist, Chapter 51, Statutes of 2002), which required the CSU to provide courses and training in gerontology for professional service delivery personnel providing services to the senior population. These professional service delivery personnel included gerontologists, nurses, physical therapists, psychologists, and social workers. Perhaps the call for a new legislative bill might extend the intent of these prior bills to include the education and training of long-term care administrators (such as assisted living administrators and/or nursing home administrators) as well as their "interprofessional team" members, described in Chapter 8.

Collaboration and partnerships are key in the successful education and training of students who will be competent and skilled in working for, and with, the elderly residing in assisted living/residential care communities. Academic programs in gerontology, such as the one at San Francisco State University (https://pace.sfsu.edu/gerontology), have collaborated with a variety of community partners in the assisted living/residential care industry. Moreover, in working together, it is apparent that the assisted living/residential care profession is on a trajectory for significant job growth. For example, the California Assisted Living Association (CALA) website 
has an informative section on workforce development (https://caassistedliving.org/workforce/) where you will find tools and resources to help assisted living, memory care, and continuing care retirement community (CCRC) practitioners develop and sustain a workforce ready to meet these escalating needs. Whether through community partners serving as guest lecturers, internship preceptors, and/or participation in a careers-in-aging job fair, building relationships by offering student-learning opportunities can help develop the much-needed workforce in assisted living/ residential care communities.

Students interested in learning more about the assisted living/residential care profession can apply for scholarship support, through organizations like CALA, to attend a professional conference. Participation at a professional conference enables students to: (a) gain a greater understanding of the assisted living/residential care profession, (b) meet face-to-face with prospective employers online or onsite at the professional conference, (c) attend cutting-edge educational sessions and network with assisted living/residential care professionals, and (d) hear from an assisted living/residential care professional who can share strategies for navigating a career path. Furthermore, from the assisted living/residential care professional perspective, research shows building a team of quality student interns - who are then employed as staff members-leads to a high quality of life for residents.

In addition to academic courses/degrees in gerontology, state certification in assisted living/ residential care administration is imperative. Each state sets its own assisted living/residential care regulations and administers its own certification examination. Furthermore, an optional Certified Director of Assisted Living (CDAL) program may be used as a means of promoting professionalism within the senior living industry. The CDAL program is administered under the authority of the Senior Living Certification Commission (SLCC), which is part of Argentum (https://www .argentum.org/assisted-living-executive-director-certification-program).

Ongoing and continuing education for assisted living/residential care administrators and their interprofessional team members remains a cornerstone for continued excellence in elder care delivery. Mandated institutional trainings, professional continuing education, specialty care training, and disaster management trainings addressing natural disasters and the recent COVID19 pandemic represent educational areas of importance for administrators, practitioners, staff, and residents.

As noted throughout this second edition, assisted living administrators are required to stay current with state-mandated regulations and national recommendations and guidelines. Education for assisted living administrators and interprofessional team members includes state-mandated core trainings and program trainings focused on the domains of practice. These domains of practice include organizational management, human resource management, business and financial management, environmental management, and resident care management. Requirements for mandated institutional trainings in each state are described in reviews of assisted living regulations (National Center for Assisted Living, 2019) and should be a major component of required educational programs. Comprehensive information about mandated trainings is well described in Chapter 5.

Practitioners working in assisted living/residential care communities update their understanding of elder care delivery for residents through a number of formal professional continuing-education programs. Updated information for content on physical aspects of aging, such as memory care, nutrition, medication administration, management of illnesses, including heart disease and strokes, are examples of areas in which professionals regularly improve their education. Continuing-education programs are offered through professional organizations, universities, state 
licensing boards, and private companies. One example of a state-level organization is the Florida Senior Living Association (FSLA), whose annual conferences, continuing-education courses, and certification exam review questions enable assisted living administrators, practitioners, and staff to remain current through broad-based educational offerings (https://floridaseniorliving.org/). In addition, Chapter 7 offers a comprehensive review of effective practices.

Assisted living community members must remain current in understanding and management of emergencies and disasters. The COVID-19 pandemic presents a new and growing national challenge for administrators, practitioners, staff, and residents as well as for family and friends of assisted living residents. Members of assisted living communities, especially residents and staff members from at-risk demographic groups, remain at significant risk for morbidity and mortality associated with COVID-19. Current and ongoing education is critical in providing administrators and practitioners with effective practices to keep vulnerable assisted living community members safe through the diagnosis, assessment, and management of COVID-19 by offering coronavirus testing, contact tracing, quarantine, and current medical treatments. Federal and state guidelines from the Centers of Disease Control and Prevention, the U.S. Public Health Department, state and local public health departments, and public health and medical and elder organization trainings are examples of educational efforts to provide necessary information during the current healthcare crisis. Chapter 12 of this second edition offers a beneficial review of basic information on disaster-preparedness issues.

As we reflect on the second edition of Assisted Living Administration and Management: Effective Practices and Model Programs in Elder Care during what appears to be a marginalization of older Americans (especially those residing in assisted living/residential care communities) with the rise of COVID-19, we offer the following five-step call to action:

1. Call attention to the need to require education about aging, aging processes, and elder care workforce needs and its contribution to individual, family, community, and societal health and well-being.

2. Call attention to the need to cultivate collaboration and partnerships between colleges/ universities and assisted living/residential care communities to provide studentlearning opportunities such as internship placements.

3. Call attention to the need to implement effective state regulations, state certification, and continuing education in assisted living/residential care administration.

4. Call attention to the benefit of facilitating workforce development and careers-in-aging in assisted living/residential care communities to meet the growing needs of the elderly who age in place and are cared for in place.

5. Last, call attention to actions that may help to save lives of residents of assisted living/ residential care communities during the COVID-19 pandemic.

A comprehensive COVID-19 action plan to save lives of residents of California nursing homes and assisted living communities was developed by the California Advocates for Nursing Home Reform* (CANHR; https://canhrnews.com/wp-content/uploads/2020/04/CANHR_COVID-19_ Emergency_Action_Plan.pdf). CANHR's action plan includes the following details, which can be adapted and considered for implementation in other states:

\footnotetext{
* Reproduced with permission of California Advocates for Nursing Home Reform.
} 
- End the ban on visitation.

The visitation ban has harmed residents by isolating them and contributing to unmet needs and neglect. Give every resident the right to at least one support-person visitor who can visit in person until the time when full visitation rights can be restored. Establish reasonable infection control precautions for support-person visitors and provide training on safe visitation. Visitation saves lives.

- Stop COVID-19 from being introduced into long-term care facilities.

Order facilities with no known or suspected COVID-19 outbreaks to refuse admission to any outside patients who have tested positive for COVID-19. When COVID-19 enters long-term care facilities, it is highly likely it will spread and kill residents despite precautions.

- Designate facilities to provide care to COVID-19 patients after they are discharged from hospitals.

Establish COVID-19 dedicated postacute care facilities in counties and require all hospital postdischarge patients to be tested for COVID-19 and, if positive, to be transferred to such facilities. Current facility residents should not be displaced to create COVID-19-dedicated facilities; rather, available empty spaces should be used. Set strong standards for dedicated facilities. Ban operators and facilities with poor track records or histories of outbreaks or noncompliance from designation. Post information on designated facilities.

- Monitor on a daily basis facilities residents or staff who have COVID-19.

Assign an inspector to conduct daily onsite monitoring visits at each facility with residents or staff who have active COVID-19 and at facilities with poor compliance histories, to ensure infection control practices and staffing levels are safe and to sound the alarm on the need for immediate intervention if they are not.

- Deploy strike teams to intervene at facilities when residents are endangered.

At the earliest sign of a facility crisis related to COVID-19 that endangers the lives of residents, send strike teams composed of state and local health departments, local health systems, emergency medical services (EMS), the National Guard, ombudsman programs, and Centers for Medicare \& Medicaid Services (CMS) Region 9 personnel to provide emergency leadership, medical treatment, care, testing, supplies, and equipment to save the lives of residents in overburdened facilities. Each strike team should give daily public reports describing its actions, findings, resources needed, and the status of residents and staff in the facility.

- Ensure staffing is sufficient to keep residents safe.

Require all long-term care facilities to maintain safe staffing levels; to submit daily staffing reports to California Department of Public Health $(\mathrm{CDPH})$ or Community Care Licensing Division (CCLD), local health departments, ombudsman programs, and CMS; and to post the daily reports on facility, state, and local health department websites.

- Provide "hazard pay" and paid sick leave to workers so they do not need to work in multiple long-term care facilities simultaneously or need to work while sick. 
To help address understaffing and prevent cross-transmission of the virus during the pandemic, require long-term care facilities to pay double-time to health care workers during the crisis, and to provide at least 2 weeks of paid sick leave.

Restore full inspections and investigate all complaints at long-term care facilities.

Inspectors and investigators are the last line of defense for facility residents who are being mistreated, neglected, or abused. Require regulatory agencies to carry out comprehensive inspections and to investigate all complaints in accordance with the law.

- Enforce infection control standards and residents' rights.

Impose immediate sanctions on facilities that jeopardize residents' lives through poor infection control or other substandard care, illegal evictions, or other violations of the law. Direct inspectors to identify and document violations. Post inspection findings and sanctions on existing state websites.

- Mandate transparency on infection levels.

Require facilities to inform residents, families, staff members, state and local health departments, and the local long-term care ombudsman when residents or staff test positive for COVID-19 and when residents or transferred residents die due to confirmed or suspected cases of COVID19 , along with the steps the facility is taking to treat infected residents and to protect other residents. Impose strong penalties on facilities that fail to report or submit false data.

- Fully inform the public on outbreaks at long-term care facilities.

Direct state and local health departments to identify long-term care facilities that have residents or staff members with positive COVID-19 test results, to fully track and report deaths of residents at each facility or following transfers, and to publicly report and update the status of facility infections and deaths on a daily basis.

Test all staff and residents frequently.

Ensure availability of testing with rapid results at all long-term care facilities statewide. Impose strong penalties against facilities that fail to comply with testing requirements. Report facility-specific findings publicly and update data daily. Implement contact tracing procedures when a positive result is discovered.

- Ensure availability of personal protective equipment (PPE) at long-term care facilities.

Give long-term care facilities high priority for distribution of PPE.

- Enable residents to go home temporarily if they are able to do so.

Give residents who wish to return home temporarily the means to do so by expediting assistance to provide home caregivers, make testing readily available, and give residents the right to return to their long-term care facilities once the crisis recedes or if their stays at home become unsafe or unmanageable.

- Suspend nursing home and assisted living evictions.

During the COVID-19 crisis, it is nearly impossible for vulnerable people being evicted from nursing homes and assisted living facilities to find new places to live. Prevent homelessness by temporarily suspending evictions from these facilities during the pandemic. 
The five suggestions in the preceding call to action have one common denominator: this second edition of Assisted Living Administration and Management: Effective Practices and Model Programs in Elder Care. This book provides an informative and useful resource for both students and practitioners alike, as they navigate effective practices and model programs for those they serve successfully in assisted living/residential care communities.

Darlene Yee-Melichar, EdD, FGSA, FAGHE Andrea Renwanz Boyle, PhD, RN, FNAP

Cristina Flores, PhD, RN, FGSA

\section{REFERENCES}

California Integrated Elder Care and Involvement Act of 2002. https://leginfo.legislature.ca.gov/faces/ billSearchClient.xhtml?session_year=20192020\&house=Both\&author=All\&lawCode $=$ All

National Center for Assisted Living. (2019). 2019 assisted living state regulatory review. https://www .ahcancal.org/Assisted-Living/Policy/Documents/2019_reg_review.pdf 